\title{
EDITORIAL
}

\section{IN THE APRIL 2007 ISSUE OF CLINICS}

\author{
Mauricio Rocha-e-Silva, Editor
}

This April 2007 issue of CLINICS highlights a paper by Giribela et al, who measured the effects of a low-dose oral contraceptive (15 1/4 g ethinyl estradiol/60 $1 / 4 \mathrm{~g}$ gestodene) on venous endothelial function of 20 healthy young women in a prospective case control study. Venous endothelial function was evaluated at baseline and after 4 months in the oral contraceptive users group (11 women) and in a control group ( 9 women). After preconstriction of the vein with phenylephrine, dose-response curves for acetylcholine and sodium nitroprusside were constructed. These preliminary data show that a reduction, although not statistically significant, occurred in the maximum venodilation response to acetylcholine and sodium nitroprusside after 4 months of oral contraceptive use; no significant changes were detected in the control group. The authors note that further studies are necessary using the same methodology in a larger sample over a longer follow-up period to ascertain whether the reduction seen in the current study is reliably reproducible.

We also publish 11 other original articles.

Hayek et al evaluated percutaneous cryotherapy as a primary treatment option for high-risk prostate cancer patients in 21 high-risk prostate cancer patients who underwent 24 percutaneous prostate cryoablation procedures. They showed that percutaneous cryotherapy is a safe minimally invasive treatment, but with a poor PSA-free survival outcome in these high-risk prostate cancer patients. They suggest that other treatment modalities should be concomitantly used in this critical risk group.

Toledo et al evaluated the influence of physical training with or without noninvasive ventilation at 2 levels of airway pressure $\left(\mathrm{BiPAP}^{\circledR}\right)$ in 18 patients with chronic obstructive pulmonary disease performing physical training on a treadmill with or without $\mathrm{BiPAP}^{\circledR}$ for 30 minutes, 3 times a week, for 12 weeks. At identical levels of physical exercise, both groups exhibited significant improvements in dyspnea, peripheral oxygen saturation, and distance walked during the physical training, as well as respiratory

Hospital das Clínicas, São Paulo University Medical School - São Paulo/ SP, Brazil.

Email: mrsilva36@hcnet.usp.br muscle strength. Only the physical training with BiPAP group had significant improvements in heart rate, systolic blood pressure, oxygen consumption, and blood lactate, showing that physical training associated with $\mathrm{BiPAP}^{\circledR}$ enhanced the oxidative muscular capacity and could be an adjunctive recourse for physical rehabilitation in patients with chronic obstructive pulmonary disease.

Pracchia et al investigated the value of the 18Ffluorodeoxyglucose test performed with a dual-head coincident gamma camera (CGC-PET with fluorodeoxyglucose) for the staging and detection of residual tumors of 38 patients with Hodgkin lymphoma. CGC-PET with FDG was done in 18 patients for staging work-up (Group 1), and the results were compared to conventional clinical staging procedures that included computed tomography scans and bone marrow biopsy. The remaining 20 patients were evaluated with CGC-PET with fluorodeoxyglucose due to the presence of residual masses or a new lesion (Group 2). The CGC-PET with fluorodeoxyglucose test upstaged 5 of the Group 1 patients and detected more lesions (45) than conventional methods of staging (33). Of the 20 patients in Group 2, 11 had positive 18F-fluorodeoxyglucose tests, and a viable tumor was confirmed in 9 patients. In the 9 patients with negative fluorodeoxyglucose metabolic tests, the 1 -year probability of recurrence was $11.8 \%$. They concluded that the metabolic test comprising CGC-PET with fluorodeoxyglucose has a higher diagnostic accuracy than conventional methods in the staging of Hodgkin lymphoma and is a valuable noninvasive tool for the diagnosis of suspicious lesions.

Portes et al compared torque and the hamstring/quadriceps ratio of the knee of 9 athletes with, and 19 without, anterior cruciate ligament laxity using an isokinetic machine model Cybex 770. The peak torque of quadriceps and hamstrings was compared, and the hamstring/quadriceps ratio at a constant angular speed of $60^{\circ}$ per second was also compared. The main finding was that anterior cruciate ligament laxity of long distance-running athletes did not significantly alter the peak torque of flexors and of extensors or the hamstring/quadriceps ratio.

Rezende et al performed a prospective study to evaluate the profile of patients with dentoalveolar trauma assisted 
in the emergency room of the Oral and Maxillofacial Trauma and Surgery Service at the Hospital das Clínicas, São Paulo University Medical School (Brazil). The study was conducted through a questionnaire applied during the first attendance of those patients over a period of 8 months. The authors concluded that the incidence of dentoalveolar trauma decreased with age and the main etiologic factors in adult patients were traffic accidents and physical assault.

Shinjo et al translated and adapted the Bath Ankylosing Spondylitis Methodology Index - a metrological measurement for the assessment of patients with ankylosing spondylitis-to Brazilian-Portuguese, and they analyzed the applicability of the questionnaire. The Brazilian-Portuguese version of the Bath Ankylosing Spondylitis Methodology Index was achieved through a translation and back-translation process. The new Index version was administered to 25 consecutive patients with ankylosing spondylitis who met the 1984 New York criteria. The authors concluded was that there was no conflict stemming from translation and re-translation of the questionnaire, and that cross-cultural adaptation proved unnecessary. This proved that the Index is an applicable instrument for analyzing the mobility index of Brazilian patients with ankylosing spondylitis.

Bonfim et al studied the effect of hemodialysis on intra-abdominal pressure in 5 patients in an intensive care unit. Intra-abdominal pressure was measured before and after hemodialysis, maintaining the ventilatory parameters except for PEEP (positive-end expiratory pressure). It was shown that intra-abdominal pressure was significantly reduced by hemodialysis in all the 5 patients, an effect that could influence other organic systems. The reduction is related to the weight variation before and after hemodialysis, as well as to the loss of volume caused by this procedure.

Sugayama et al developed a scoring system based on clinical findings to assist pediatricians in the diagnosis of Williams syndrome and to delineate when the fluorescent in-situ hybridization test to detect the microdeletion at $7 q 11.23$ may be needed. The fluorescent in-situ hybridization test was performed on 20 patients presenting clinical features suggestive of Williams syndrome. Eleven studies were selected from the literature in which there were 2 groups: patients with positive or negative fluorescent insitu hybridization tests. Forty-two clinical characteristics were compared to those reported in the literature to determine which ones were associated with the affected patients (ie, those bearing genomic deletions) using meta-analysis. The scoring system was based on clinical findings and their significant associations with patients with positive fluorescent in-situ hybridization tests. The authors concluded that the scoring system might enable physicians to differentiate between those individuals who can be reliably diagnosed as having Williams syndrome solely from the clini- cal findings and those who need to undergo fluorescent insitu hybridization testing for a correct diagnosis.

Oliveira et al investigated the biological behavior of classical and atypical osteoblastomas in comparison to osteosarcomas. Based on histological parameters, 30 osteoblastomas were subclassified as classical osteoblastomas (23/30) or atypical osteoblastoma (high cellularity, prominent blue osteoid, and epithelioid osteoblasts-7/30). Comparative immunohistochemical and clinical analysis was performed in 17 cases of patients with high-grade osteosarcoma. Formalin-fixed, paraffin-embedded archival tissue was immunostained for p53 and proliferation cell nuclear antigen. Tumors with positive p53 stain underwent molecular analyses for fragments of exon 10. These results validate atypical osteoblastoma as an entity, with p53 and proliferation cell nuclear antigen immunoexpression being closer to that of osteosarcoma than of classical osteoblastoma. Proliferating cell nuclear antigen labeling index and p53 may be useful predictors of recurrence.

Castilho et al systematically analyzed the efficacy of chemonucleolysis in the treatment of lumbar disc herniation through a meta-analysis of 22 clinical trials selected from 3 electronic databases (The Cochrane Controlled Trials Register, MEDLINE, and EMBASE). Data were analyzed with the software, STATA, using the Meta command. The authors concluded that chemonucleolysis with chymopapain was superior to placebo and was as effective as collagenase in the treatment of lumbar disc prolapse. Results for studies comparing chemonucleolysis with surgery were heterogeneous, making it difficult to interpret the summary measure of effect.

Cabrita et al prospectively compared 2 methods of treatment of chronic infection in hip arthroplasties-with or without an antibiotic-loaded cement spacer-in 68 patients (30 with spacer, 38 without spacer) and found that the use of the spacer provides better infection control with good functional results and is superior to treatment without a spacer.

We also publish 2 reviews-one on surfactant proteins and their relevance in the development of neonatal pulmonary disease and one on urinary and sexual manifestations in HTLV-I-infected patients-and 3 case reports.

In the February 2007 issue, CLINICS published a paper entitled "Changes in joint kinematics in children with cerebral palsy while walking with and without a floor reaction ankle-foot orthosis" by PRG Lucareli et al. Authors recognize that they mistakenly omitted reference to the fact that the research which is the object of this article was performed at the "Associação de Assistência à Criança Deficiente, São Paulo, Brazil". A discussion is under progress concerning complete authorship. This article will be fully rectified as soon as all parties involved reach an agreement. 